\title{
Distribution of LIM domain kinase 1 in the olfactory bulb, cerebral cortex, hippocampus, and cerebellum of the App/PS ${ }^{+/-}$mice
}

\author{
L. An ${ }^{1,2}$, J. Liu' ${ }^{1}$, W.W. Li ${ }^{1}$, Y.J. Shi ${ }^{1}$, X.H. Lin ${ }^{2}$, D.M. Yu' ${ }^{1}$, J.B. Deng ${ }^{1}$ and \\ X.Q. $\operatorname{Ren}^{2}$ \\ ${ }^{1}$ Institute of Neurobiology, College of Life Science, Henan University, \\ Kaifeng, Henan, China \\ ${ }^{2}$ Translational Medicine Center, Huaihe Hospital, Henan University, \\ Kaifeng, Henan, China \\ Corresponding author: J.B. Deng \\ E-mail: jinbo_deng2005@163.com \\ Genet. Mol. Res. 14 (4): 17244-17251 (2015) \\ Received August 16, 2015 \\ Accepted October 18, 2015 \\ Published December 16, 2015 \\ DOI http://dx.doi.org/10.4238/2015.December.16.24
}

\begin{abstract}
LIM domain kinase 1 (LIMK1), an actin-binding kinase, can phosphorylate and inactivate its substrates, and can regulate long-term memory and synaptic plasticity. Both $\beta$-amyloid precursor protein (App) and presenilin (PS) are functional degeneration factors during early neuronal development, and are considered as potential factors that contribute to the development of Alzheimer's disease (AD). However, hardly any information is available about the distribution and expression of LIMK1. Thus, using the App and PS deficient mice, the role of LIMK1 was demonstrated in the absence of App and PS. Our results showed that LIMK1 was present in the nerve fiber layer and external plexiform layer of the olfactory bulb, as well as in the mitral cells and Purkinje cells of the cerebellum in App and PS deficient mice. Additionally, LIMK1 was concentrated in the granule cell layer of the olfactory bulb and cerebellum and LIMK1 positive cells were located in the CA1 region of the hippocampus. Our study indicates that there is a connection between LIMK1 and AD in the mouse model of AD. This might explain neurological
\end{abstract}


problems such as cerebellar ataxia, impaired long-term memory, and impaired synaptic plasticity observed in AD.

Key words: Olfactory bulb; Cerebellum; Granule cell; Alzheimer's disease

\section{INTRODUCTION}

$\beta$-amyloid $(A \beta)$, a product of $\beta$-amyloid precursor protein (APP), is aggregated into oligomers, fibrils and plaques, which is a central phenomenon in the molecular pathogenesis of Alzheimer's disease (AD) (Vorobyeva et al, 2014; Rudy et al., 2015). AD is a progressive neurodegenerative disorder leading to memory loss and cognitive deficits (Piau et al., 2011; Rudy et al., 2015). Presenilin (PS) is part of the $y$-secretase complex, and it is responsible for $A D$ with an earlier age of onset. Its enzymatic activity is responsible for hydrolyzing proteins at aspartic acid molecules, and PS regulates the signaling regulating differentiation and development (Collins et al., 2015). Cognitive impairments and synaptic loss in animal models of $A D$ suggest that the presence of amyloid- $\beta$ is associated with $A D$ in transgenic mouse models (Sahlholm et al., 2015). The double transgenic mouse model with amyloid- $\beta /$ presenilin deposition (App/PS ${ }^{+/}$) is widely used to study AD (Weitz et al., 2014). Members of the LIM kinase family play an important role in actin dynamics through the regulation of cofilin via phosphorylation and thus inactivation. LIM domain kinase 1 (LIMK1) is mostly expressed in neurons with mature synapses, and is involved in inhibiting bone morphogenetic protein 6 during apoptosis and AD through its protective effects in App/PS ${ }^{+/}$transgenic mice (Sun et al., 2014).

The mammalian olfactory bulb is mainly composed of the mitral cells (MC), tufted cells, granule cells, and periglomerular cells. The pathways formed by these cells are responsible for smelling and its regulation in the olfactory bulb, for example, the mitral cells form projections in the olfactory bulb (Nagayama et al., 2014). The mammalian cerebral cortex develops in an "inside-out" pattern during development (Sekine et al., 2012). The hippocampus is a specific region that is necessary for memory formation (Tastet et al., 2012). Cerebellar gray matter, which can be divided into the molecular layer, Purkinje cell layer, and granule cell layer, forms the surface of the cerebellum. Interestingly, the excitation of Purkinje cells leads to movements whose timing is more closely matched with training intervals (Lee et al., 2015). Previous studies showed that LIMKs are involved in learning, memory, neuronal migration, the function of neuronal circuits, and smelling (Yang et al., 2004; Todorovski et al., 2015). These studies suggest that LIMKs might contribute to $A D$ in the aging brain.

To investigate this issue, we examined the expression and distribution of LIMK1 in the brain. Interestingly, LIMK1 was associated with the layered structures of the olfactory bulb, cerebral cortex, hippocampus, and cerebellum in the App/PS ${ }^{+/}$mouse model of AD.

\section{MATERIAL AND METHODS}

\section{Animals}

C57BL/6J mice and App/PS ${ }^{+/-}$transgenic mice (the strain number D000268) were purchased from Model Animal Research Center of Nanjing University and used in the experiments. All animal experiments were performed according to the guidelines for the care and use of laboratory animals of Henan University. 


\section{Reagents and antibodies}

Phosphate buffer saline (PBS) and antibody dilution buffer were purchased from Gibco (USA); paraformaldehyde (PFA, Sangon biotech, China) was stored in our lab. The primary antibody rabbit anti-LIMK1 and the secondary antibody Alexa Fluor 488-conjugated goat antirabbit were purchased from Invitrogen (USA). We purchased 4',6-diamidino-2-phenylindole (DAPI) from Millipore (USA).

\section{Immunohistochemistry}

Mice with an age of approximately 270 days were used for this study. Pelltobarbitalum Natricum was used to anesthetize mice and 4\% PFA was used to perfuse the animals for 10 min. Their brains were fixed with 4\% PFA in PBS for $1 \mathrm{~h}$ at room temperature, and the fixed olfactory bulb, cerebral cortex, hippocampus, and cerebellum were incubated in rabbit antiLIMK1 (1:500) overnight at $4^{\circ} \mathrm{C}$. Then the slices were incubated in the secondary antibody Alexa Fluor 488-conjugated goat anti-rabbit (1:300) for $2 \mathrm{~h}$ at room temperature. Finally, the nuclei were stained with DAPI $(1: 1000)$ for $5 \mathrm{~min}$ at room temperature. Stained slices were analyzed using the FV1000 fluorescence microscope (Olympus).

\section{RESULTS}

\section{Expression of LIMK1 in four key parts of the mouse brain}

Relevant studies have shown that LIMK1 plays a significant role in the function of neuronal circuits, synaptic plasticity, learning, and neuronal migration (Chai et al., 2009; Piccioli and Littleton, 2014). As a cellular protein with Ser/Thr and Tyr protein kinase activities in multiple cell types, LIMK1 was found in cells of the olfactory bulb, cerebral cortex, hippocampus, and cerebellum in wild-type (WT) mice and in App/PS ${ }^{+/}$mice (Figure 1); therefore, immunofluorescent signals were detectable throughout four key parts of the mouse brain. Strong signals were found in the marginal zone of the olfactory bulb, in the cerebral cortex, in the cornu ammonis (CA) regions of the hippocampus, dentate gyrus (DG) of the hippocampus, and gray matter of the cerebellum. Additionally, LIMK1 was found in the neurons of four key parts of the mouse brain. Interestingly, the expression of LIMK1 was decreased in the deeper layers of the olfactory bulb and cerebellum in WT mice compared with those in the transgenic App/PS ${ }^{+/-}$mouse model of AD.

\section{LIMK1 is expressed in the granule cell layer of the olfactory bulb in the mouse model of AD, but not in WT mice}

The olfactory bulb is compose of multiple layers with a variety of cells, and the role of these cell types is not yet fully understood regarding the function of neuronal circuits (Nagayama et al., 2014). To investigate the expression and distribution of LIMK1, the olfactory bulb was immunolabeled with an anti-LIMK1 antibody. Figure 2 shows that strong fluorescent signals were found in the olfactory bulb of the App/PS ${ }^{+/-}$mouse model of AD. LIMK1 was found in the nerve fiber layer (NFL) and external plexiform layer (EPL) in both WT mice and the App/PS ${ }^{+/-}$mouse model of $A D$, while in the WT mice, no signal was visible in the inner granule cell layer (GRL) apart 
from a few cells. In the GRL, LIMK1 immunostaining was different between the two groups. These results indicate that LIMK1 might play an important role in the neuronal function of the olfactory bulb and the development of AD.

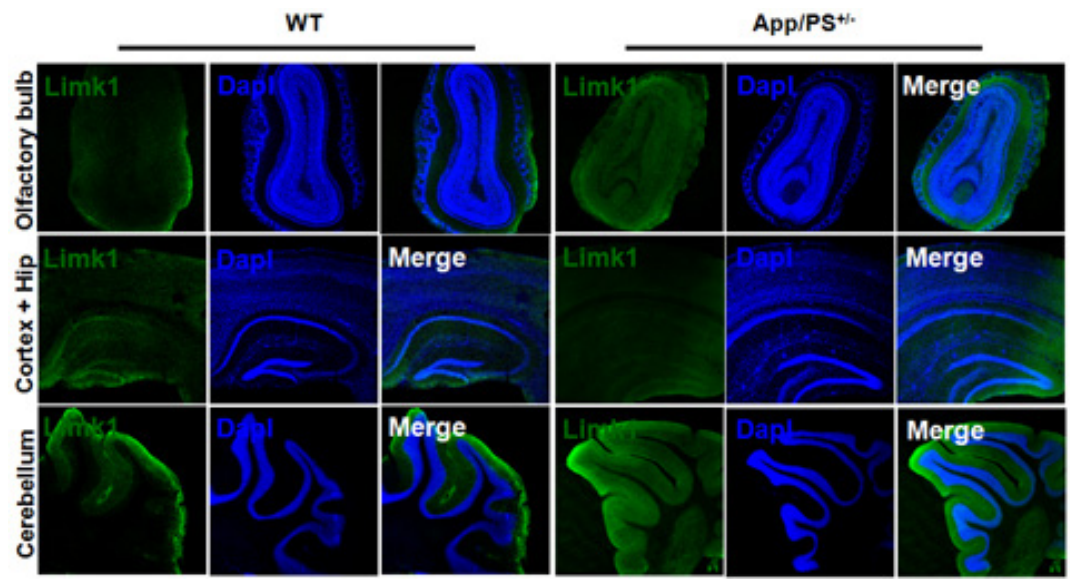

Figure 1. Expression of LIMK1 in four key parts of the mouse brain. Immunostaining for LIM domain kinase 1 (LIMKI; green) and 4',6-diamidino-2-phenylindole (DAPI; blue) staining in sections from wild-type (WT) mice and the App/ $\mathrm{PS}^{+/-}$mouse model of Alzheimer's disease. LIMK1 (left panels) immunohistochemical signal is present in the olfactory bulb, cerebral cortex, hippocampus, and cerebellum of WT mice and App/PS ${ }^{+/}$transgenic mice. DAPI (middle panels) staining reveals the nuclei of cells forming layers.
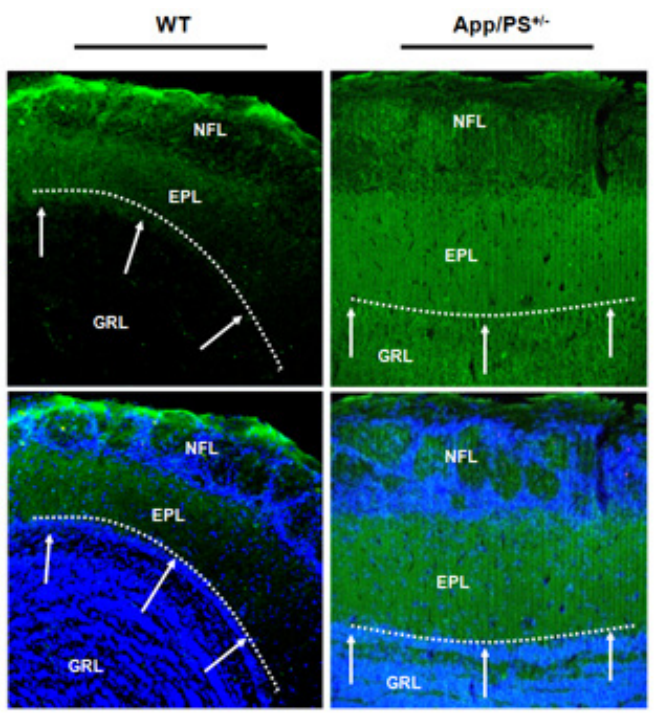

Figure 2. Expression of LIMK1 in the olfactory bulb at a high magnification. Immunohistochemical signal of LIM domain kinase 1 (LIMK1; upper panels) is present in the nerve fiber layer (NFL) and external plexiform layer (EPL) of the olfactory bulb in both wild-type (WT) mice and the App/PS ${ }^{+-}$mouse model of Alzheimer's disease, and in the granule cell layer (GRL) in the App/PS ${ }^{+/-}$mice. Furthermore, LIMK1 is located in the nucleus of granule cells and the GRL. Merged images (lower panels) revealed that LIMK1 was located in the nuclei and layers of the olfactory bulb (arrows and dotted line show the border of the GRL and $\mathrm{EPL}$ ). 


\section{Expression of LIMK1 in the cerebral cortex and hippocampus in WT mice and the mouse model of $A D$}

LIMK1 is increasingly recognized for its role in spine morphology and synaptic function (Meng et al., 2002). To investigate the expression and distribution of LIMK1, the cerebral cortex and hippocampus were immunostained with an anti-LIMK1 antibody, and nuclei were stained with DAPI. Figure 3 shows that strong immunofluorescent signals were found in the cerebral cortex. LIMK1 was detected in most granule cells, glia, and mature neurons of the cerebral cortex in the marginal zone (MZ), cortical plate (CP/upper $\mathrm{CP}$ ), and ventricular zone (VZ) in both control mice and the $A p p / P S^{+/-}$mouse model of $A D$. Interestingly, a thinner pyramidal cell layer was identified in the CA1 region of the App/PS ${ }^{+/-}$mouse model of $A D$ compared with that of WT mice, and a few neurons were detected outside of the CA1 pyramidal layer, which were immunopositive for LIMK1 (arrows in Figure 4). We found no significant differences in the distribution of LIMK1 in the DG and CA3 between the mouse groups. These results suggest that LIMK1 might play an important role in neurodegeneration, as the hippocampus is known for its function in learning and memory.

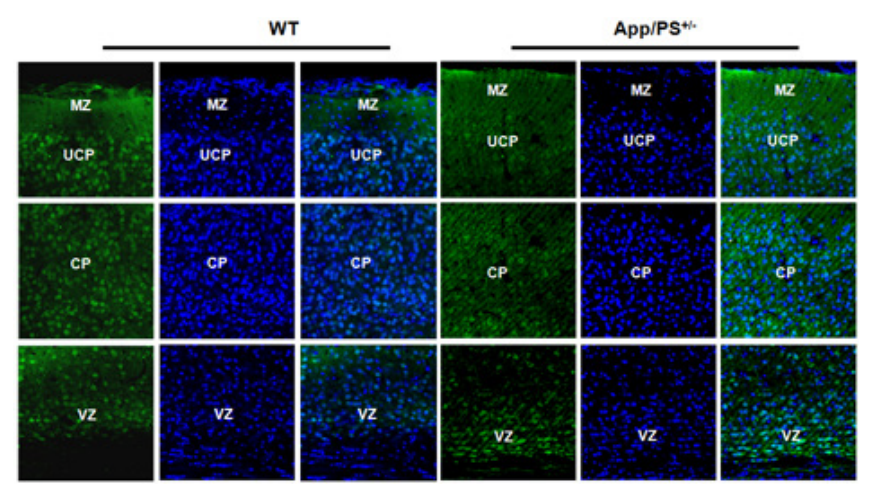

Figure 3. Distribution of LIMK1 in the cerebral cortex at a high magnification. LIM domain kinase 1 (LIMK1; green) and 4',6-diamidino-2-phenylindole (DAPI; blue) staining in the marginal zone (MZ), cortical plate (CP), upper cortical plate (UCP), and ventricular zone (VZ).
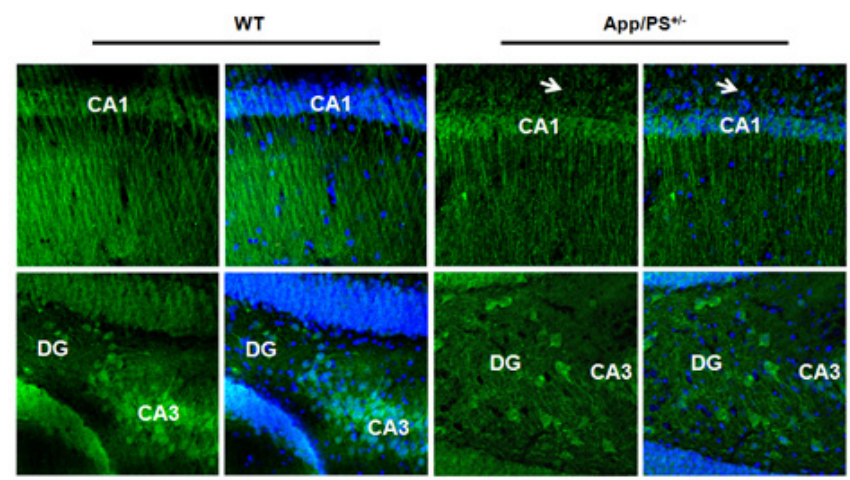

Figure 4. Distribution of LIMK1 in the hippocampus at a high magnification. Immunostaining for LIM domain kinase 1 (LIMK1; green) in sections from wild-type (WT) mice and the App/PS ${ }^{+-}$mouse model of Alzheimer's disease. LIMK1 immunopositive signal is located in all regions of the hippocampus, and is increased in the nuclei of neurons in the CA regions. Arrow shows a neuron outside of the CA1. 


\section{More LIMK1 is localized in the granule cell layer of the cerebellum in the mouse model of AD than in WT mice}

The cerebellar cortex is composed of the molecular cell layer (MC), Purkinje cell layer (PC), and granule cell layer (GC), and the inhibition of Purkinje cells lead to impaired learning and thus circuit changes in the cerebellar nuclei (Lee et al., 2015). To investigate LIMK1 expression and distribution in the cerebellum, we immunolabeled the cerebellum with an anti-LIMK1 antibody, and stained the nuclei with DAPI. Figure 5 shows that strong fluorescent signals were found in the cerebellum. Similar to WT mice, LIMK1 was found in the MC in the App/PS ${ }^{+/}$mouse model of AD. In the $P C$, a very weak signal was identified in the $A p p / P^{+/-}$mouse model of $A D$, while the signal was strong in WT mice, and LIMK1 was concentrated in the cytoplasm, but not the nuclei of Purkinje cells (WT in Figure 5). More interestingly, a low level of LIMK1 positive signal was identified in the inner GC in WT mice, nevertheless, in App/PS ${ }^{+/-}$mice, LIMK1 expression co-localized with DAPI in the deeper layers of GC (Figure 5). Therefore, LIMK1 might play a key role in the signaling of Purkinje cells in the cerebellum. These results suggest that AD might misregulate Purkinje cells and the excitability of granule cells during neuronal degeneration.

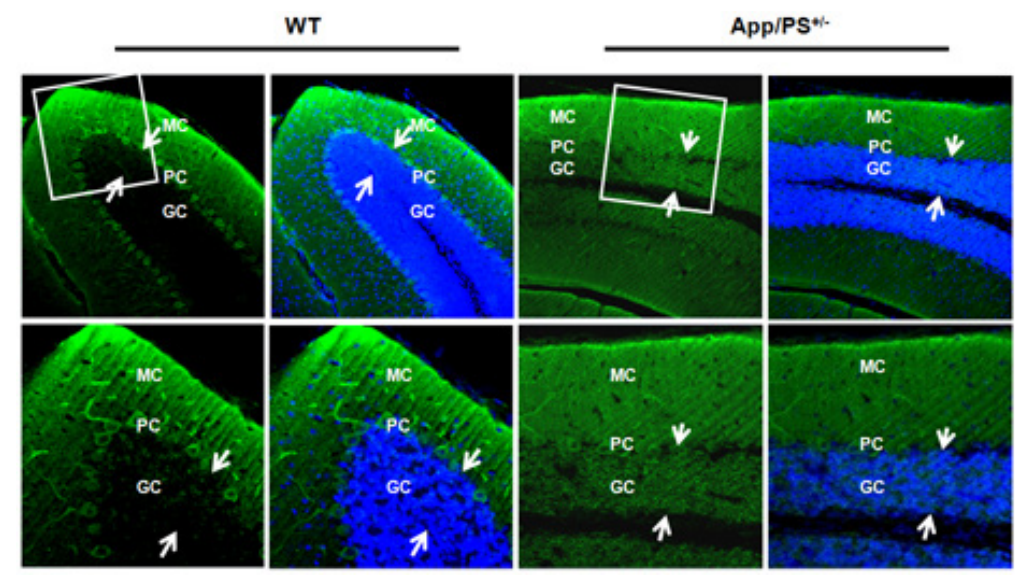

Figure 5. Expression of LIMK1 in the cerebellum at a high magnification. Immunostaining for LIM domain kinase 1 (LIMK1; green) and 4',6-diamidino-2-phenylindole (DAPI; blue) staining in sections from wild-type (WT) mice and the App/PS ${ }^{+/-}$mouse model of Alzheimer's disease. Immunopositive signal for LIMK1 is mainly located in the mitral cells (MC) and Purkinje cells (PC) of the cerebellum. In WT mice, there is no immunofluorescent signal in the granule cells (arrows are indicating the granule cell layer).

\section{DISCUSSION}

In addition to its function in actin dynamics, LIMK1 is inhibited by $A \beta$, and this inhibition can alter the structure of cytoskeleton and lead to cognitive dysfunction (Maloney et al., 2005). Therefore, we studied the relationship between LIMK1 and AD in the mouse brain. AD is the most common form of dementia in patients, and it is characterized by the accumulation of $A \beta$, which leads to the impairment of learning and memory and thus cognitive deficits (Rudy et al., 2015).

Previous studies indicated that LIMK1 regulate the phosphorylation of its substrates and synaptic function in the brain. In LIMK1 knockout mice, cofilin phosphorylation and excitatory synaptic function in the CA1 region of the hippocampus are severely impaired (Meng et al., 2004). 
Identification of olfactory dysfunction in preclinical and early stages of AD is a potentially useful method to detect the disease (Attems et al., 2014; Masurkar and Devanand, 2014), and similar to LIMK1, several factors regulate the function of cortical neurons. In transgenic mouse models of dementia, the cerebellar pathology of dementia is maintained (Franco et al., 2011; Purushothuman et al., 2015). Our results showed that LIMK1 was detected in the NFL and EPL of the olfactory bulb in WT mice and the App/PS ${ }^{+/-}$mouse model of AD. In addition, LIMK1 is expressed in the granule cells of the olfactory bulb and cerebellum in the App/PS ${ }^{+/}$mouse model of $A D$. Revealing the interactions between LIMK1 and AD might help to understand the fundamentals of central nervous system degeneration and olfactory dysfunction, which is an early biomarker of neurodegenerative disorders and disease progression. In this study, we identified LIMK1 expression in the GRL of the olfactory bulb in the App/PS ${ }^{+-}$mouse model of AD. However, the immunofluorescent signal of LIMK1 was weak in the GC layer of the olfactory bulb and cerebellum in WT mice. A few immunopositive cells were detected outside of the CA1 pyramidal layer of the hippocampus in the App/PS $\mathrm{PS}^{+/-}$mouse model of $A D$. LIMK1 is involved in the development of PS/Aß-induced $A D$ and the regulation of long-term memory and synaptic plasticity in mice (Meng et al., 2002; Sun et al., 2014).

The redundant App, which is responsible for $A D$, is characterized by severe neurodegeneration leading to impaired learning and memory, and animals with App overexpression are highly susceptible to AD (Ling et al., 2003; Weitz et al., 2014; Collins et al., 2015; Cummings et al., 2015; Sahlholm et al., 2015). In mice, the defect of LIMK1 causes deficits in the late phase of long-term potentiation and in memory. In this study, we revealed that LIMK1, a critical factor for normal neuronal function, plays a role in brain development, and induces AD through the spreading of granule cells and few projective cells (Figures 2, 4, 5). In addition, we noticed that the distribution of LIMK1 is homogeneous in the olfactory bulb, cerebellum, cerebral cortex, and hippocampus. These results showed that LIMK1 plays a central role in the activation in a spatiotemporal manner of APP and PS. Furthermore, LIMK1 was expressed in the GRL and mitral cells in the App/PS ${ }^{+/-}$ mouse model of AD. Our results showed that LIMK1 expression was decreased in the inner granule cells beneath the PC. As LIMK1 might be a useful candidate for studying memory (Wang et al., 2013; Wolf et al., 2014), the present results suggest that LIMK1 and AD are connected from the cytoplasm to the nucleus, which might cause several degenerative diseases.

Collectively, these results indicate that following conclusion, App/PS was present in the brain of $A D$ mice, and the reaction was associated with LIMK1. Distribution of LIMK1 was strongly related to the development of $A D$. Relationship of $A D$ and LIMK1 will be investigated in further studies.

Based on the present study, we have shown that in the App/PS-induced AD, tissues responsible for cognitive functions are affected by neurodegeneration, and the altered expression of LIMK1 in granule cells and other cell types is involved the development of AD. Furthermore, during the process of AD, LIMK1 was distributed diffusely and maintained the process. To conclude, our findings suggest that LIMK1 regulates the development of AD via the central neuronal system of the brain.

\section{Conflicts of interest}

The authors declare no conflict of interest.

\section{ACKNOWLEDGMENTS}

Research supported by the Startup Project of Henan University Science Research (\#B2014120) and National Natural Science Foundation of China (\#31070952, \#U1204311 and \#U1204809). 


\section{REFERENCES}

Attems J, Walker L and Jellinger KA (2014). Olfactory bulb involvement in neurodegenerative diseases. Acta Neuropathol. 127: 459-475.

Chai X, Förster E, Zhao S, Bock HH, et al. (2009). Reelin stabilizes the actin cytoskeleton of neuronal processes by inducing n-cofilin phosphorylation at serine3. J. Neurosci. 29: 288-299.

Collins JM, King AE, Woodhouse A, Kirkcaldie MT, et al. (2015). The effect of focal brain injury on beta-amyloid plaque deposition, inflammation and synapses in the APP/PS1 mouse model of Alzheimer's disease. Exp. Neurol. 267: 219-229.

Cummings JL, Isaacson RS, Schmitt FA and Velting DM (2015). A practical algorithm for managing Alzheimer's disease: what, when, and why? Ann. Clin. Transl. Neurol. 2: 307-323.

Franco SJ, Martinez-Garay I, Gil-Sanz C, Harkins-Perry SR, et al. (2011). Reelin regulates cadherin function via Dab1/Rap1 to control neuronal migration and lamination in the neocortex. Neuron 69: 482-497.

Lee KH, Mathews PJ, Reeves AM, Choe KY, et al. (2015). Circuit mechanisms underlying motor memory formation in the cerebellum. Neuron 86: 529-540.

Ling Y, Morgan K and Kalsheker N (2003). Amyloid precursor protein (APP) and the biology of proteolytic processing: relevance to Alzheimer's disease. Int. J. Biochem. Cell Biol. 35: 1505-1535.

Maloney MT, Minamide LS, Kinley AW, Boyle JA, et al. (2005). Beta-secretase-cleaved amyloid precursor protein accumulates at actin inclusions induced in neurons by stress or amyloid beta: a feedforward mechanism for Alzheimer's disease. $J$. Neurosci. 25: 11313-11321.

Masurkar AV and Devanand DP (2014). Olfactory dysfunction in the elderly: basic circuitry and alterations with normal aging and Alzheimer's disease. Curr. Geriatr. Rep. 3: 91-100.

Meng Y, Zhang Y, Tregoubov V, Janus C, et al. (2002). Abnormal spine morphology and enhanced LTP in LIMK-1 knockout mice. Neuron 35: 121-133.

Meng Y, Takahashi H, Meng J, Zhang Y, et al. (2004). Regulation of ADF/cofilin phosphorylation and synaptic function by LIMkinase. Neuropharmacology 47: 746-754.

Nagayama S, Homma R and Imamura F (2014). Neuronal organization of olfactory bulb circuits. Front. Neural Circuits 8: 98.

Piau A, Nourhashémi F, Hein C, Caillaud C, et al. (2011). Progress in the development of new drugs in Alzheimer's disease. J. Nutr. Health Aging 15: 45-57.

Piccioli ZD and Littleton JT (2014). Retrograde BMP signaling modulates rapid activity-dependent synaptic growth via presynaptic LIM kinase regulation of cofilin. J. Neurosci. 34: 4371-4381.

Purushothuman S, Johnstone DM, Nandasena C, Eersel J, et al. (2015). Near infrared light mitigates cerebellar pathology in transgenic mouse models of dementia. Neurosci. Lett. 591: 155-159.

Rudy CC, Hunsberger HC, Weitzner DS and Reed MN (2015). The role of the tripartite glutamatergic synapse in the pathophysiology of Alzheimer's disease. Aging Dis. 6: 131-148.

Sahlholm K, Liao F, Holtzman DM, Xu J, et al. (2015). Sigma-2 receptor binding is decreased in female, but not male, APP/PS1 mice. Biochem. Biophys. Res. Commun. 460: 439-445.

Sekine K, Kawauchi T, Kubo K, Honda T, et al. (2012). Reelin controls neuronal positioning by promoting cell-matrix adhesion via inside-out activation of integrin a5ß1. Neuron. 76: 353-369.

Sun L, Guo C, Wang T, Li X, et al. (2014). LIMK1 is involved in the protective effects of bone morphogenetic protein 6 against amyloid- $\beta$-induced neurotoxicity in rat hippocampal neurons. J. Alzheimers Dis. 42: 543-554.

Tastet J, Vourc'h P, Laumonnier F, Vallée B, et al. (2012). LIMK2d, a truncated isoform of Lim kinase 2 regulates neurite growth in absence of the LIM kinase domain. Biochem. Biophys. Res. Commun. 420: 247-252.

Todorovski Z, Asrar S, Liu J, Saw NM, et al. (2015) LIMK1 regulates long-term memory and synaptic plasticity via the transcriptional factor CREB. Mol. Cell Biol. 35: 1316-1328.

Vorobyeva AG, Lee R, Miller S, Longen C, et al. (2014). Cyclopamine modulates Y-secretase-mediated cleavage of amyloid precursor protein by altering its subcellular trafficking and lysosomal degradation. J. Biol. Chem. 289: 33258-33274.

Yang EJ, Yoon JH, Min DS and Chung KC (2004) LIM kinase 1 activates cAMP-responsive element-binding protein during the neuronal differentiation of immortalized hippocampal progenitor cells. J. Biol. Chem. 279: 8903-8910.

Wang Y, Dong Q, Xu XF, Feng X, et al. (2013). Phosphorylation of cofilin regulates extinction of conditioned aversive memory via AMPAR trafficking. J. Neurosci. 33: 6423-6433.

Weitz TM, Gate D, Rezai-Zadeh K and Town T (2014). MyD88 is dispensable for cerebral amyloidosis and neuroinflammation in APP/PS1 transgenic mice. Am. J. Pathol. 184: 2855-2861.

Wolf M, Zimmermann AM, Görlich A, Gurniak CB, et al. (2015) ADF/Cofilin controls synaptic actin dynamics and regulates synaptic vesicle mobilization and exocytosis. Cereb. Cortex. 25: 2863-2875. 